\title{
Role of Chaplygin gas as geometrical dark energy in anisotropic brane gravity
}

\author{
Malihe Heydari-Fard* and Hamid R. Sepangi ${ }^{\dagger}$ \\ Department of Physics, Shahid Beheshti University, Evin, Tehran 19839, Iran
}

January 9, 2019

\begin{abstract}
We consider an anisotropic brane-world model with Bianchi type I and V geometry, without mirror symmetry or any form of junction conditions. The generalized Chaplygin gas, which interpolates between a high density relativistic era and a non-relativistic matter phase, is a popular candidate for the present accelerated expansion of the universe. Considering the generalized Chaplygin gas as a geometrical dark energy, we obtain the general solutions in an exact parametric form for both Bianchi type I and V space-times. Finally, we study the behavior of the observationally important parameters such as the shear, anisotropic and deceleration parameter in this model.
\end{abstract}

PACS numbers: 04.50.-h, 04.20.-q, 04.20.Jb

\section{Introduction}

From a large number of observational evidence, the observable universe is presently undergoing an accelerated expansion. As an alternative to both the cosmological constant and quintessence, it is also possible to explain the acceleration of the universe by introducing a cosmic fluid component with an exotic equation of state, called Chaplygin gas. The Chaplygin gas model describes a transition from a universe filled with dust-like matter to an accelerated expanding stage. The Generalized Chaplygin Gas (GCG) model, introduced in [1] and elaborated in [2], is described by a perfect fluid obeying an exotic equation of state

$$
p_{c h}=-\frac{A}{\rho_{c h}^{\alpha}},
$$

where $A$ is a positive constant and $0<\alpha \leq 1$. The original Chaplygin gas corresponds to $\alpha=1$. However, recent analyses based on the latest of type-Ia Supernovae data have yielded rather surprising results, namely that $\alpha>1$ and that there is a degeneracy between the GCG and xCDM models in the form of a phantom-like energy component [30]. An attractive feature of the model is that it can naturally explain both dark energy and dark matter [3]. Within the framework of FriedmannRobertson-Walker cosmology, this equation of state leads, after having inserted into the relativistic energy conservation equation, to an energy density evolving as

$$
\rho_{c h}=\left[A+\frac{C}{a^{3(1+\alpha)}}\right]^{\frac{1}{1+\alpha}},
$$

*email: m.heydarifard@mail.sbu.ac.ir

†email: hr-sepangi@sbu.ac.ir 
where $a$ is the scale-factor of the universe and $C$ is an integration constant which should be positive for a well-defined $\rho_{c h}$ at all times. Hence, we see that $\rho_{c h} \sim a^{-3}$ at early times, that is, $\rho_{c h}$ behaves as matter while at late times it behaves like a cosmological constant $\rho_{c h} \sim$ constant. The Chaplygin gas appears in the stabilization of branes in Schwarzschild anti-de Sitter (AdS) black hole bulks as a critical theory at the horizon [4] and in the string analysis of black holes in three dimensions [5]. The Chaplygin gas also appears as an effective fluid associated with $d$-branes [6] and can also be derived from the Born-Infeld action [7]. An interesting range of models have been found to be consistent with the SNe Ia data [8], CMB experiments [9] and other observational data [10]. The cosmological implications of the Chaplygin gas model have been intensively investigated in the literature $[11,12,13]$.

The idea that our familiar 4-dimensional $(4 D)$ space-time is a hypersurface (brane) in a $5 \mathrm{D}$ spacetime (bulk) $[14,15,16]$ has been under detailed elaboration during the last decade. According to this brane-world scenario, all matter and gauge interactions reside on the brane, while gravity can propagate in the 5D space-time. Several brane-world cosmologies have been proposed in the context of the Randall-Sundrum (RS) formulations [15], defined in a 5-dimensional anti-de Sitter space-time. The dynamics of these models feature boundary terms in the action and sometimes mirror symmetry, such that bulk gravitational waves interfere with the brane-world motion. This usually comes together with junction conditions producing an algebraic relationship between the extrinsic curvature and the confined matter $[17,18]$. The consequence is that the Friedman equation acquires an additional term which is proportional to the square of energy density of the confined matter field $[19,20]$. This term was initially considered as a possible solution to the accelerated expansion of the universe. However, soon it was realized to be incompatible with the big bang nucleosynthesis, requiring additional fixes $[20]$.

Brane-world scenarios under more general conditions and still compatible with the brane-world program have also been rather extensively studied over the past decade where it has been shown that it is possible to find a richer set of cosmological solutions in accordance with the current observations [21]. Under these conditions, without using $Z_{2}$ symmetry or without postulating any junction condition, Friedman equation is modified by a geometrical term which is defined in terms of the extrinsic curvature, leading to a geometrical interpretation for dark energy [22]. There has also been arguments concerning the uniqueness of the junction conditions. Indeed, other forms of junction conditions exist, so that different conditions may lead to different physical results [18]. Furthermore, these conditions cannot be used when more than one non-compact extra dimension is involved. Against this background, an interesting higher-dimensional model was introduced in [23] where particles are trapped on a $4 \mathrm{D}$ hypersurface by the action of a confining potential. The dynamics of test particles confined to a brane by the action of such potential at the classical and quantum levels were studied in [24]. In [25], the same brane-world model was studied, offering a geometrical explanation for the accelerated expansion of the universe. A geometrical explanation for the generalized Chaplygin gas was considered in [26] along the same line. In this paper, we consider an anisotropic brane-world model with Bianchi type I and V geometries filled with a geometrical Chaplygin gas. The behavior of the observationally important physical quantities is studied in this scenario.

\section{The model}

The embedding of the brane-world in the bulk plays an essential role in the covariant formulation of the brane-world gravity, because it tells us how the Einstein-Hilbert dynamics of the bulk is transferred to the brane. However, there are many different ways to embed a manifold into another, classified as local, global, isometric, conformal, rigid, deformable, analytic or differentiable. The choice of one or other depends on what the embedded manifold is supposed to do.

Let us present a brief review of the model proposed in [22]. Consider the background manifold $\bar{V}_{4}$ isometrically embedded in a pseudo-Riemannian manifold $V_{m}$ by the map $\mathcal{Y}: \bar{V}_{4} \rightarrow V_{m}$, with $m=4+n$ components $\mathcal{Y}^{A}$ such that

$$
\mathcal{G}_{A B} \mathcal{Y}_{, \mu}^{A} \mathcal{Y}_{, \nu}^{B}=\bar{g}_{\mu \nu}, \quad \mathcal{G}_{A B} \mathcal{Y}_{, \mu}^{A} \overline{\mathcal{N}}_{a}^{B}=0, \quad \mathcal{G}_{A B} \overline{\mathcal{N}}_{a}^{A} \overline{\mathcal{N}}_{b}^{B}=\bar{g}_{a b}
$$


where $\mathcal{G}_{A B}\left(\bar{g}_{\mu \nu}\right)$ is the metric of the bulk (brane) space $V_{m}\left(\bar{V}_{4}\right)$ in arbitrary coordinates, $\left\{\mathcal{Y}^{A}\right\}\left(\left\{x^{\mu}\right\}\right)$ is the basis of the bulk (brane) and $\mathcal{N}_{a}^{A}$ are $n$ normal unit vectors orthogonal to the brane. According to Nash [27], we may continuously perturb $\bar{V}_{4}$ along a normal direction in the bulk, to obtain another submanifold of the same bulk, provided the embedding functions remain regular. Perturbation of $\bar{V}_{4}$ in a sufficiently small neighborhood of the brane along an arbitrary transverse direction $\xi$ is given by

$$
\mathcal{Z}^{A}=\mathcal{Y}^{A}+\left(\mathcal{L}_{\xi} \mathcal{Y}\right)^{A}, \quad \mathcal{N}^{A}=\overline{\mathcal{N}}^{A}+\left(\mathcal{L}_{\xi} \overline{\mathcal{N}}\right)^{A}=\overline{\mathcal{N}}^{A}
$$

where $\mathcal{L}$ represents the Lie derivative and $\xi^{a}(a=1,2, \ldots, n)$ is a small parameter along $\mathcal{N}_{a}^{A}$, parameterizing the extra noncompact dimensions. By choosing $\xi$ orthogonal to the brane, we ensure gauge independency and have perturbations of the embedding along a single orthogonal extra direction $\overline{\mathcal{N}}_{a}$ giving local coordinates of the perturbed brane as

$$
\mathcal{Z}_{, \mu}^{A}=\mathcal{Y}_{, \mu}^{A}+\xi^{a} \overline{\mathcal{N}}_{a, \mu}^{A}
$$

The above assumptions lead to the embedding equations of the perturbed geometry

$$
\mathcal{G}_{A B} \mathcal{Z}_{, \mu}^{A} \mathcal{Z}_{, \nu}^{B}=g_{\mu \nu}, \quad \mathcal{G}_{A B} \mathcal{Z}_{, \mu}^{A} \mathcal{N}_{a}^{B}=g_{\mu a}, \quad \mathcal{G}_{A B} \mathcal{N}_{a}^{A} \mathcal{N}_{b}^{B}=g_{a b}
$$

From these equations it follows that

$$
g^{\mu \nu} \mathcal{Z}_{, \mu}^{A} \mathcal{Z}_{, \nu}^{B}=\mathcal{G}^{A B}-g^{a b} \mathcal{N}_{a}^{A} \mathcal{N}_{b}^{B},
$$

and also the components of the perturbed geometry

$$
\begin{gathered}
g_{\mu \nu}=\mathcal{G}_{A B} \mathcal{Z}_{, \mu}^{A} \mathcal{Z}_{, \nu}^{B}=\bar{g}_{\mu \nu}-2 \xi^{a} \bar{K}_{\mu \nu a}+\xi^{a} \xi^{b}\left[\bar{g}^{\alpha \beta} \bar{K}_{\mu \alpha a} \bar{K}_{\nu \beta b}+g^{c d} \bar{A}_{\mu c a} \bar{A}_{\nu d b}\right], \\
g_{\mu b}=\mathcal{G}_{A B} \mathcal{Z}_{, \mu}^{A} \mathcal{N}_{b}^{B}=\xi^{a} A_{\mu a b}, \\
g_{a b}=\mathcal{G}_{A B} \mathcal{N}_{a}^{A} \mathcal{N}_{b}^{B}=\bar{g}_{a b}, \\
K_{\mu \nu a}=-\mathcal{G}_{A B} \mathcal{N}_{a, \mu}^{A} \mathcal{Z}_{, \nu}^{B}=\bar{K}_{\mu \nu a}-\xi^{b}\left[\bar{g}^{\alpha \beta} \bar{K}_{\mu \alpha a} \bar{K}_{\nu \beta b}+g^{c d} \bar{A}_{\mu c a} \bar{A}_{\nu d b}\right] \\
A_{\mu a b}=\mathcal{G}_{A B} \mathcal{N}_{a, \mu}^{A} \mathcal{N}_{b}^{B}=\bar{A}_{\mu a b},
\end{gathered}
$$

where $A_{\mu a b}$ represents the twisting vector fields and $\bar{K}_{\mu \nu a}, K_{\mu \nu a}$ represent the extrinsic curvature of the original and perturbed brane respectively. Comparing equations (8) and (11), we obtain

$$
K_{\mu \nu a}=-\frac{1}{2} \frac{\partial g_{\mu \nu}}{\partial \xi^{a}}
$$

which is the generalized York's relation and shows how the extrinsic curvature propagates as a result of the propagation of the metric in the direction of extra dimensions. The components of the Riemann tensor of the bulk written in the embedding vielbein $\left\{\mathcal{Z}_{, \alpha}^{A}, \mathcal{N}_{a}^{A}\right\}$, lead to the Gauss-Codazzi and Ricci equations, respectively [28]

$$
\begin{gathered}
R_{\alpha \beta \gamma \delta}=2 g^{a b} K_{\alpha[\gamma a} K_{\delta] \beta b}+\mathcal{R}_{A B C D} \mathcal{Z}_{, \alpha}^{A} \mathcal{Z}_{, \beta}^{B} \mathcal{Z}_{, \gamma}^{C} \mathcal{Z}_{, \delta}^{D}, \\
2 K_{\alpha[\gamma c ; \delta]}=2 g^{a b} A_{[\gamma a c} K_{\delta] \alpha b}+\mathcal{R}_{A B C D} \mathcal{Z}_{, \alpha}^{A} \mathcal{N}_{c}^{B} \mathcal{Z}_{, \gamma}^{C} \mathcal{Z}_{, \delta}^{D}, \\
2 A_{[\gamma a b ; \delta]}=-2 g^{c d} A_{[\gamma c a} A_{\delta] d b}-g^{c d} K_{[\gamma c a} K_{\delta] d b}-\mathcal{R}_{A B C D} \mathcal{N}_{a}^{A} \mathcal{N}_{b}^{B} \mathcal{Z}_{, \gamma}^{C} \mathcal{Z}_{, \delta}^{D},
\end{gathered}
$$


where $\mathcal{R}_{A B C D}$ and $R_{\alpha \beta \gamma \delta}$ are the Riemann tensors for the bulk and the perturbed brane respectively. Contracting the Gauss equation (14) on $\alpha$ and $\gamma$ we find

$$
R_{\mu \nu}=\left(K_{\mu \alpha c} K_{\nu}^{\alpha c}-K_{c} K_{\mu \nu}^{c}\right)+\mathcal{R}_{A B} \mathcal{Z}_{, \mu}^{A} \mathcal{Z}_{, \nu}^{B}-g^{a b} \mathcal{R}_{A B C D} \mathcal{N}_{a}^{A} \mathcal{Z}_{, \mu}^{B} \mathcal{Z}_{, \nu}^{C} \mathcal{N}_{b}^{D}
$$

A further contraction then gives the Ricci scalar

$$
R=\left(K_{\mu \nu a} K^{\mu \nu a}-K_{a} K^{a}\right)+\mathcal{R}-2 g^{a b} \mathcal{R}_{A B} \mathcal{N}_{a}^{A} \mathcal{N}_{b}^{B} .
$$

Therefore, the Einstein-Hilbert action for the bulk geometry in $m$-dimensions can be written as

$$
\begin{aligned}
S & =\frac{1}{2 \alpha_{*}} \int\left(\mathcal{R}-2 \Lambda^{(b)}\right) \sqrt{\mathcal{G}} d^{m} x \\
& =\frac{1}{2 \alpha_{*}} \int\left[R-\left(K_{\mu \nu a} K^{\mu \nu a}-K_{a} K^{a}\right)+2 g^{a b} \mathcal{R}_{A B} \mathcal{N}_{a}^{A} \mathcal{N}_{b}^{B}-2 \Lambda^{(b)}\right] \sqrt{\mathcal{G}} d^{m} x .
\end{aligned}
$$

Variation of the action with respect to $\mathcal{G}_{A B}$ gives the Einstein field equations in the bulk

$$
\mathcal{R}_{A B}-\frac{1}{2} \mathcal{R} \mathcal{G}_{A B}=\alpha_{*} T_{A B}^{*}-\Lambda^{(b)} \mathcal{G}_{A B},
$$

where $\alpha_{*}=\frac{1}{M_{*}^{m-2}}$ and $\Lambda^{(b)}$ is the cosmological constant of the bulk space. The vielbein components of the energy-momentum tensor are given by

$$
T_{\mu \nu}^{*}=T_{A B}^{*} \mathcal{Z}_{, \mu}^{A} \mathcal{Z}_{, \nu}^{B}, \quad T_{\mu a}^{*}=T_{A B}^{*} \mathcal{Z}_{, \mu}^{A} \mathcal{N}_{a}^{B}, \quad T_{a b}^{*}=T_{A B}^{*} \mathcal{N}_{a}^{A} \mathcal{N}_{b}^{B} .
$$

The tangent components follow from the contractions of equation (20) with $\mathcal{Z}_{, \mu}^{A} \mathcal{Z}_{, \nu}^{B}$. After using equations (17) and (18) we obtain the "gravi-tensor" equation

$$
R_{\mu \nu}-\frac{1}{2} R g_{\mu \nu}-Q_{\mu \nu}+g^{a d} \mathcal{R}_{A B C D} \mathcal{N}_{a}^{A} \mathcal{Z}_{, \mu}^{B} \mathcal{Z}_{, \nu}^{C} \mathcal{N}_{d}^{D}-g^{a b} \mathcal{R}_{A B} \mathcal{N}_{a}^{A} \mathcal{N}_{b}^{B} g_{\mu \nu}=\alpha_{*} T_{\mu \nu}^{*}-\Lambda^{(b)} g_{\mu \nu}
$$

where

$$
Q_{\mu \nu}=g^{a b}\left(K_{\mu a}^{\rho} K_{\rho \nu b}-K_{a} K_{\mu \nu b}\right)-\frac{1}{2}\left(K_{\alpha \beta a} K^{\alpha \beta a}-K_{a} K^{a}\right) g_{\mu \nu}
$$

By a direct calculation we can see that the extra term $Q_{\mu \nu}$ is an independently conserved quantity. On the other hand, the trace of the Codazzi equation gives the "gravi-vector" equation

$$
\begin{aligned}
K_{\mu a ; \rho}^{\rho} & -\left(g^{\mu \nu} K_{\mu \nu a}\right)_{, \mu}-\left(A_{\rho c a} K_{\mu}^{\rho c}-A_{\mu c a} g^{\alpha \beta} K_{\alpha \beta}^{c}\right)+2 g^{c d} \mathcal{R}_{A B C D} \mathcal{N}_{a}^{A} \mathcal{N}_{c}^{B} \mathcal{Z}_{, \mu}^{C} \mathcal{N}_{d}^{D} \\
& =\alpha_{*}\left(T_{\mu a}^{*}-\frac{1}{n+2} T^{*} g_{\mu a}\right)+\frac{2}{n+2} \Lambda^{(b)} g_{\mu a} .
\end{aligned}
$$

Finally, the "gravi-scalar" equation is obtained from equations (18) and (20)

$$
R-K_{\mu \nu a} K^{\mu \nu a}+K_{a} K^{a}=-2 \alpha_{*}\left(g^{a b} T_{a b}^{*}-\frac{n-1}{n+2} T^{*}\right)+2 \Lambda^{(b)}\left[n-\frac{(n-1)(n+4)}{(n+2)}\right] .
$$

In its most general form, without assuming extra dimensional matter, the confinement hypothesis states that the only non-vanishing components of $T_{A B}$ are the tangent components $T_{\mu \nu}$ representing the confined sources. Therefore we set

$$
\alpha_{*} T_{\mu \nu}^{*}=8 \pi G T_{\mu \nu}, \quad \alpha_{*} T_{\mu a}^{*}=0, \quad \alpha_{*} T_{a b}^{*}=0 .
$$

Equations (22)-(25) represent the most general equations of motion of a brane-world, compatible with the differentiable embedding in a $m$-dimensional bulk defined by the Einstein equations. Clearly, the usual Einstein equations are recovered when all elements of the extrinsic geometry are removed from those equations.

The geometrical approach considered here is based on three basic postulates, namely, the confinement of the standard gauge interactions to the brane, the existence of quantum gravity in the bulk and finally, the embedding of the brane-world. All other model dependent properties such as warped metric, mirror symmetries, radion or extra scalar fields, fine tuning parameters like the tension of the brane and the choice of a junction condition are left out as much as possible in our calculations [21]. 


\section{$3 \quad$ Field equations and observational parameters in anisotropic brane}

In what follows, we will investigate the influence of the extrinsic curvature terms on the anisotropic universe described by Bianchi type I and V geometries. From a formal point of view these two geometries are described by the line element

$$
d s^{2}=-d t^{2}+a_{1}^{2}(t) d x^{2}+a_{2}^{2}(t) e^{-2 \beta x} d y^{2}+a_{3}^{2}(t) e^{-2 \beta x} d z^{2},
$$

where $a_{i}(t), i=1,2,3$ are the expansion factors in different spatial directions. The metric for the Bianchi type I geometry corresponds to the case $\beta=0$, while for the Bianchi type $\mathrm{V}$ we have $\beta=1$. Let us define the following variables [29]

$$
v=\prod_{i=1}^{3} a_{i}, \quad H_{i}=\frac{\dot{a}_{i}}{a_{i}}, i=1,2,3, \quad 3 H=\sum_{i=1}^{3} H_{i}, \quad \Delta H_{i}=H_{i}-H, i=1,2,3 .
$$

In above equations, $v$ is the volume scale factor, $H_{i}, i=1,2,3$ are the directional Hubble parameters, and $H$ is the mean Hubble parameter. The physical quantities of observational importance in cosmology are the expansion scalar $\Theta$, the mean anisotropy parameter $A$, the shear scalar parameter $\sigma^{2}$, and the deceleration parameter $q$, which are defined according to

$$
\Theta=3 H, \quad 3 A=\sum_{i=1}^{3}\left(\frac{\Delta H_{i}}{H}\right)^{2}, \quad \sigma^{2}=\frac{1}{2} \sigma_{i j} \sigma^{i j}=\frac{1}{2} \sum_{i=1}^{3} H_{i}^{2}-3 H^{2}, \quad q=\frac{d}{d t}\left(\frac{1}{H}\right)-1 .
$$

The sign of the deceleration parameter indicates how the universe expands. A positive sign for $q$ corresponds to the standard decelerating models whereas a negative sign indicates an accelerating expansion at late times. We note that $A=0$ for an isotropic expansion.

Let us assume that the confined source on the brane is a perfect fluid with a linear barotropic equation of state, namely $p=(\gamma-1) \rho$ with $1 \leq \gamma \leq 2$. In this paper we restrict our analysis to a five-dimensional bulk with a constant curvature characterized by the Riemann tensor

$$
\mathcal{R}_{A B C D}=k_{*}\left(\mathcal{G}_{A C} \mathcal{G}_{B D}-\mathcal{G}_{A D} \mathcal{G}_{B C}\right)
$$

where $k_{*}$ denotes the bulk constant curvature. In the flat case $k_{*}=0$ and in the de Sitter and anti-de Sitter cases we may write $k_{*}= \pm \frac{\Lambda^{(b)}}{6}$ respectively. Assuming $g_{55}=1$ and using the Gauss-Codazzi equations, we obtain

$$
\begin{gathered}
R_{\alpha \beta \gamma \delta}=\left(K_{\alpha \gamma} K_{\beta \delta}-K_{\alpha \delta} K_{\beta \gamma}\right)+k_{*}\left(g_{\alpha \gamma} g_{\beta \delta}-g_{\alpha \delta} g_{\beta \gamma}\right), \\
K_{\alpha[\beta ; \gamma]}=0 .
\end{gathered}
$$

The equations of motion derived in the previous section can be obtained directly from equations (31) and (32). The result is Einstein equations as modified by the presence of the extrinsic curvature

$$
G_{\mu \nu}=8 \pi G T_{\mu \nu}-\Lambda g_{\mu \nu}+Q_{\mu \nu}
$$

where $\Lambda=-3 k_{*}+\Lambda^{(b)}$ is the effective cosmological constant in four dimensions with $Q_{\mu \nu}$ being a completely geometrical quantity given by

$$
Q_{\mu \nu}=\left(K K_{\mu \nu}-K_{\mu \alpha} K_{\nu}^{\alpha}\right)+\frac{1}{2}\left(K_{\alpha \beta} K^{\alpha \beta}-K^{2}\right) g_{\mu \nu},
$$

where $K=g^{\mu \nu} K_{\mu \nu}$. Using the York relation

$$
K_{\mu \nu a}=-\frac{1}{2} \frac{\partial g_{\mu \nu}}{\partial \xi^{a}},
$$


we realize that in a diagonal metric, $K_{\mu \nu a}$ is diagonal. After separating the spatial components, the Codazzi equations reduce to (here $\alpha, \beta, \gamma, \sigma=1,2,3$ )

$$
\begin{gathered}
K_{\gamma a, \sigma}^{\alpha}+K_{\gamma a}^{\beta} \Gamma_{\beta \sigma}^{\alpha}=K_{\sigma a, \gamma}^{\alpha}+K_{\sigma a}^{\beta} \Gamma_{\beta \gamma}^{\alpha}, \\
K_{\gamma a, 0}^{\alpha}+\frac{\dot{a}_{i}}{a_{i}} K_{\gamma a}^{\alpha}=\frac{\dot{a}_{i}}{a_{i}} \delta_{\gamma}^{\alpha} K_{0 a}^{0}, \quad i=1,2,3 .
\end{gathered}
$$

The first equation gives $K^{1}{ }_{1 a, \sigma}=0$ for $\sigma \neq 1$, since $K_{1 a}^{1}$ does not depend on the spatial coordinates. Repeating the same procedure for $\alpha, \gamma=i, i=2,3$, we obtain $K_{2 a, \sigma}^{2}=0$ for $\sigma \neq 2$ and $K_{3 a, \sigma}^{3}=0$ for $\sigma \neq 3$. This shows that $K^{1}{ }_{1 a}, K^{2}{ }_{2 a}$ and $K^{3}{ }_{3 a}$ are functions of $t$ and the choice $K_{1 a}^{1}=K_{2 a}^{2}=$ $K^{3}{ }_{3 a}=b_{a}(t)$, where $b_{a}(t)$ are arbitrary functions of $t$, would simplify our analysis. Now from the second equation we obtain

$$
\dot{b_{a}}+\frac{\dot{a}_{i}}{a_{i}} b_{a}=\frac{\dot{a}_{i}}{a_{i}} K_{0 a}^{0}, \quad i=1,2,3 .
$$

Summing equations (38) we find

$$
K_{00 a}=-\left(\frac{3 \dot{b}_{a} v}{\dot{v}}+b_{a}\right) .
$$

For $\mu, \nu=1,2,3$ we obtain

$$
K_{\mu \nu a}=b_{a} g_{\mu \nu}
$$

Note that in equation (33) we have considered a five-dimensional bulk ( $m=5)$, thus the functions $b_{a}$ $(a=1,2, \ldots, m-4)$ reduce to only one function, namely $b_{1}$. Denoting $b_{1}=b, \theta=\frac{\dot{b}}{b}$ and $\Theta=\frac{\dot{v}}{v}$, we find from equation (34) that

$$
Q_{\mu \nu}=-3 b^{2}\left(\frac{2 \theta}{\Theta}+1\right) g_{\mu \nu}, \quad \mu, \nu=1,2,3, \quad Q_{00}=3 b^{2} .
$$

As we have noted before, $Q_{\mu \nu}$ is an independently conserved quantity, that is $Q_{; \nu}^{\mu \nu}=0$, suggesting an analogy with the energy momentum of an uncoupled non-conventional energy source. We see that solution (41) depends on the arbitrary function $b(t)$. To find the dynamical role of this function and to compare the compatibility of such geometrical model with the present experimental data, we assume $Q_{\mu \nu}$ to be a conserved energy-momentum tensor and take the GCG as an example

$$
Q_{\mu \nu} \equiv \frac{1}{8 \pi G}\left[\left(\rho_{c h}+p_{c h}\right) u_{\mu} u_{\nu}+p_{c h} g_{\mu \nu}\right], \quad p_{c h}=-\frac{A}{\rho_{c h}^{\alpha}},
$$

where $A$ and $\alpha$ are positive constants. Comparing $Q_{\mu \nu}, \mu, \nu=1,2,3$ and $Q_{00}$ from equation (42) with the components of $Q_{\mu \nu}$ and $Q_{00}$ given by equation (41), we obtain

$$
p_{c h}=-\frac{3 b^{2}}{8 \pi G}\left(\frac{2 \theta}{\Theta}+1\right), \quad \rho_{c h}=\frac{3 b^{2}}{8 \pi G} .
$$

Use of the above equations leads to an equation for $b(t)$

$$
\left(\frac{2 v \dot{b}}{\dot{v} b}+1\right)=A\left(\frac{3 b^{2}}{8 \pi G}\right)^{-(1+\alpha)},
$$

for which the solution is

$$
b(t)=\left(\frac{8 \pi G}{3}\right)^{\frac{1}{2}}\left[A+\frac{C}{v^{(1+\alpha)}}\right]^{\frac{1}{2(1+\alpha)}},
$$


where $C$ is an integration constant. Using equation (43) and this solution, the energy density of the GCG becomes

$$
\rho_{c h}=\rho_{c h_{0}}\left[A_{s}+\frac{\left(1-A_{s}\right)}{v^{(1+\alpha)}}\right]^{\frac{1}{(1+\alpha)}}
$$

where $\rho_{c h_{0}}$ is the GCG density at the present time, $A_{s}=A \rho_{c h_{0}}^{-(1+\alpha)}$ is a dimensionless quantity related to the speed of sound for the GCG today, $v_{s}^{2}=\alpha A \rho_{c h_{0}}^{-(1+\alpha)}$ and $C=\rho_{c h_{0}}^{(1+\alpha)}-A$. From equation (46), we see that for $A_{s}=0$ the GCG behaves like matter, whereas for $A_{s}=1$ it behaves as a cosmological constant and when $0<A_{s}<1$, the model predicts a behavior as that between a matter phase in the past and a negative dark energy regime at late times. This particular behavior of the GCG inspired some authors to propose a unified scheme for the cosmological "dark sector," an interesting idea which has been considered in many different contexts.

Now, using the geometrical energy density for $Q_{\mu \nu}$, the field equations on the anisotropic brane become

$$
\begin{gathered}
3 \dot{H}+\sum_{i=1}^{3} H_{i}^{2}=\Lambda-4 \pi G \rho_{0}(3 \gamma-2) v^{-\gamma}+8 \pi G \rho_{c h_{0}}\left[A_{s}+\frac{\left(1-A_{s}\right)}{v^{(1+\alpha)}}\right]^{\frac{-\alpha}{1+\alpha}}\left[A_{s}-\frac{\left(1-A_{s}\right)}{2 v^{(1+\alpha)}}\right], \\
\frac{1}{v} \frac{d}{d t}(v H)=2 \beta^{2} v^{-2 / 3}+\Lambda-4 \pi G \rho_{0}(\gamma-2) v^{-\gamma}+8 \pi G \rho_{c h_{0}}\left[A_{s}+\frac{\left(1-A_{s}\right)}{v^{(1+\alpha)}}\right]^{\frac{-\alpha}{1+\alpha}}\left[A_{s}+\frac{\left(1-A_{s}\right)}{2 v^{(1+\alpha)}}\right] .
\end{gathered}
$$

For $\beta=0$ we obtain the field equations for Bianchi type I geometry, while $\beta=1$ gives the Bianchi type $V$ equations on the anisotropic brane. Using the relation $H=\frac{\dot{v}}{3 v}$, we can rewrite equation (48) in the form

$$
\ddot{v}=6 \beta^{2} v^{1 / 3}+3 \Lambda v-12 \pi G \rho_{0}(\gamma-2) v^{1-\gamma}+24 \pi G v \rho_{c h_{0}}\left[A_{s}+\frac{\left(1-A_{s}\right)}{v^{(1+\alpha)}}\right]^{\frac{-\alpha}{1+\alpha}}\left[A_{s}+\frac{\left(1-A_{s}\right)}{2 v^{(1+\alpha)}}\right] .
$$

The general solution of equation (49) becomes

$$
t-t_{0}=\int \frac{d v}{\sqrt{9 \beta^{2} v^{4 / 3}+3 \Lambda v^{2}+24 \pi G \rho_{0} v^{2-\gamma}+f(v)+C}}
$$

where

$$
\begin{aligned}
f(v) & =\frac{24 \pi G \rho_{c h_{0}}}{2+\alpha} v\left(1+\frac{A_{s} v^{1+\alpha}}{1-A_{s}}\right)^{\frac{\alpha}{1+\alpha}}\left[1+A_{s}\left(v^{1+\alpha}-1\right)\right]^{\frac{-\alpha}{1+\alpha}} \\
& \times\left[2 A_{s} v^{1+\alpha}{ }_{2} F_{1}\left(\frac{2+\alpha}{1+\alpha}, \frac{\alpha}{1+\alpha} \frac{3+2 \alpha}{1+\alpha}, \frac{A_{s} v^{1+\alpha}}{A_{s}-1}\right)\right. \\
& \left.+(2+\alpha)\left(1-A_{s}\right)_{2} F_{1}\left(\frac{1}{1+\alpha}, \frac{\alpha}{1+\alpha} \frac{2+\alpha}{1+\alpha}, \frac{A_{s} v^{1+\alpha}}{A_{s}-1}\right)\right],
\end{aligned}
$$

and $C$ is a constant of integration. The time variation of the physically important parameters described above in the exact parametric form, with $v$ taken as a parameter, is given by

$$
\begin{gathered}
\Theta=3 H=\frac{\sqrt{9 \beta^{2} v^{4 / 3}+3 \Lambda v^{2}+24 \pi G \rho_{0} v^{2-\gamma}+f(v)+C}}{v}, \\
a_{i}=a_{0 i} v^{1 / 3} \exp \left[\int \frac{h_{i} d v}{v \sqrt{9 \beta^{2} v^{4 / 3}+3 \Lambda v^{2}+24 \pi G \rho_{0} v^{2-\gamma}+f(v)+C}}\right], \quad i=1,2,3,
\end{gathered}
$$




$$
\begin{gathered}
A=\frac{3 h^{2}}{9 \beta^{2} v^{4 / 3}+3 \Lambda v^{2}+24 \pi G \rho_{0} v^{2-\gamma}+f(v)+C}, \\
\sigma^{2}=\frac{h^{2}}{2 v^{2}}, \\
q=2-\frac{9 \beta^{2} v^{4 / 3}+3 \Lambda v^{2}+12 \pi G \rho_{0}(2-\gamma) v^{2-\gamma}+24 \pi G \rho_{c h_{0}} v^{2}\left[A_{s}+\frac{\left(1-A_{s}\right)}{v^{(1+\alpha)}}\right]^{\frac{-\alpha}{1+\alpha}}\left[A_{s}+\frac{\left(1-A_{s}\right)}{2 v^{(1+\alpha)}}\right]}{3 \beta^{2} v^{4 / 3}+\Lambda v^{2}+8 \pi G \rho_{0} v^{2-\gamma}+\frac{1}{3} f(v)+C},
\end{gathered}
$$

where $h_{i}, i=1,2,3$ are constants of integration and $h^{2}=\sum_{i=1}^{3} h_{i}^{2}$. The GCG provides an interesting candidate for the present accelerated expansion of the universe without resorting to an effective cosmological constant. To this end we consider $\Lambda=0$ and show that, within the context of the present model, the geometrical Chaplygin gas can be used to account for the accelerated expansion of the universe. Therefore for $\Lambda=0$ and $\alpha=1$, the dynamics of the Bianchi type I universe is controlled by the Chaplygin gas equation of state parameter $A_{s}$. In this case, the expansion, scalar factor, anisotropy, shear and deceleration parameters are respectively given by

$$
\begin{gathered}
\Theta=3 H=\frac{\sqrt{24 \pi G \rho_{0} v^{2-\gamma}+24 \pi G \rho_{c h_{0}} v^{2}\left[A_{s}+\left(1-A_{s}\right) v^{-2}\right]^{1 / 2}+C}}{v}, \\
a_{i}=a_{0 i} v^{1 / 3} \exp \left[\int \frac{h_{i} d v}{\sqrt{24 \pi G \rho_{0} v^{4-\gamma}+24 \pi G \rho_{c h_{0}} v^{4}\left[A_{s}+\left(1-A_{s}\right) v^{-2}\right]^{1 / 2}+C v^{2}}}\right], \quad i=1,2,3, \\
A=\frac{3 h^{2}}{24 \pi G \rho_{0} v^{2-\gamma}+24 \pi G \rho_{c h} v^{2}\left[A_{s}+\left(1-A_{s}\right) v^{-2}\right]^{1 / 2}+C}, \\
q=2-\frac{\sigma^{2}=\frac{h^{2}}{2 v^{2}},}{12 \pi G \rho_{0}(2-\gamma) v^{2-\gamma}+24 \pi G \rho_{c h_{0}} v^{2}\left[A_{s}+\frac{\left(1-A_{s}\right)}{v^{2}}\right]^{-1 / 2}\left[A_{s}+\frac{\left(1-A_{s}\right)}{2 v^{2}}\right]} \\
8 \pi G \rho_{0} v^{2-\gamma}+8 \pi G \rho_{c h_{0}} v^{2}\left[A_{s}+\left(1-A_{s}\right) v^{-2}\right]^{1 / 2}+C
\end{gathered}
$$

In figure 1 we present the dynamics of the deceleration parameter for different values of $A_{s}$ and for $\gamma=\frac{4}{3}$ and $\alpha=1$. This behavior is much dependent on the range of the values that $A_{s}$ can take. For having an accelerating universe, the value of $A_{s}$ should lie in the range $0 \leq A_{s} \leq 1$. In the initial stage the evolution of the Bianchi type I brane universe is non-inflationary, but in the late time limit the brane universe ends in an accelerating stage.

For a better understanding of the behavior of the mean anisotropy parameter, let us consider it as a function of the volume scale factor

$$
A(v)=\frac{3 h^{2}}{9 \beta^{2} v^{4 / 3}+3 \Lambda v^{2}+24 \pi G \rho_{0} v^{2-\gamma}+f(v)+C},
$$

where $f(v)$ is defined by equation (51). The behavior of the anisotropy parameter at the initial state depends on the values of $\alpha$ and $A_{s}$. For original Chaplygin gas with $\alpha=1$, we obtain an accelerating universe when $0 \leq A_{s} \leq 1$. From equation (62), in the limit $v \rightarrow 0$ and taking $0 \leq A_{s} \leq 1$, we find that the initial state is always anisotropic $A(v) \neq 0$. The behavior of the mean anisotropy parameter of the Bianchi type I and V geometries is illustrated, for $\alpha=1$ and different values of $A_{s}$, in figure 2 . 

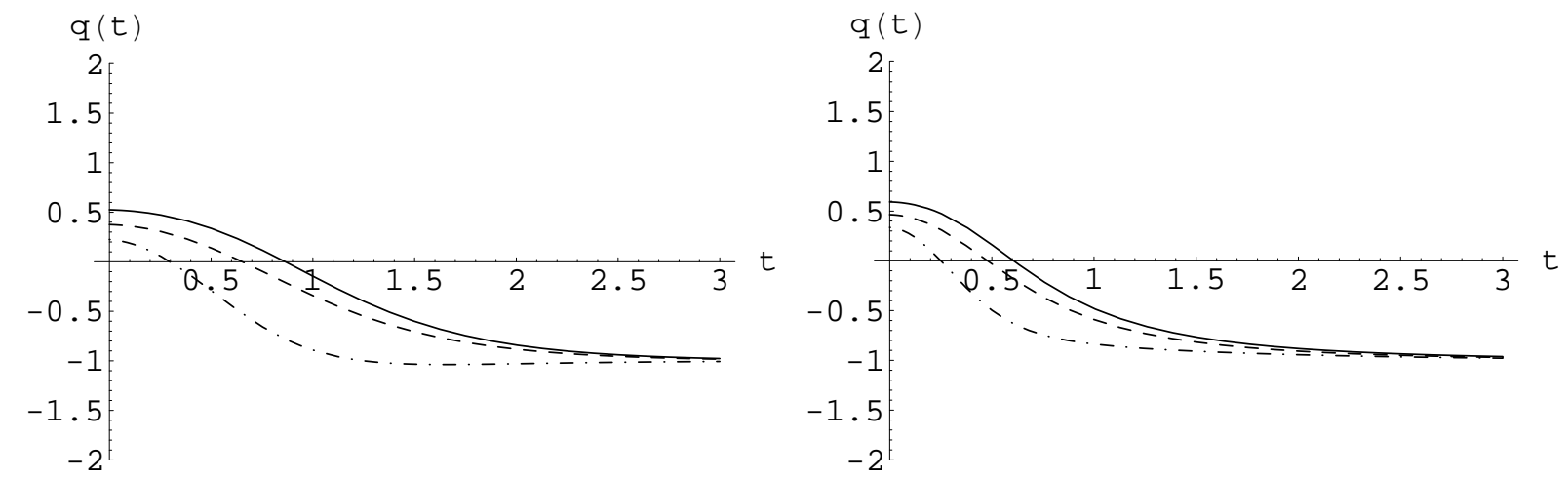

Figure 1: Left, deceleration parameter $q$ for the geometrical Chaplygin gas filled Bianchi type I brane universe as a function of time and right, the same parameter in the Bianchi type $\mathrm{V}$ brane universe for $\gamma=4 / 3, \alpha=1$ and $A_{s}=0.3$ (solid line), $A_{s}=0.5$ (dashed line), $A_{s}=0.8$ (dot-dashed line) with $\Lambda=0$.
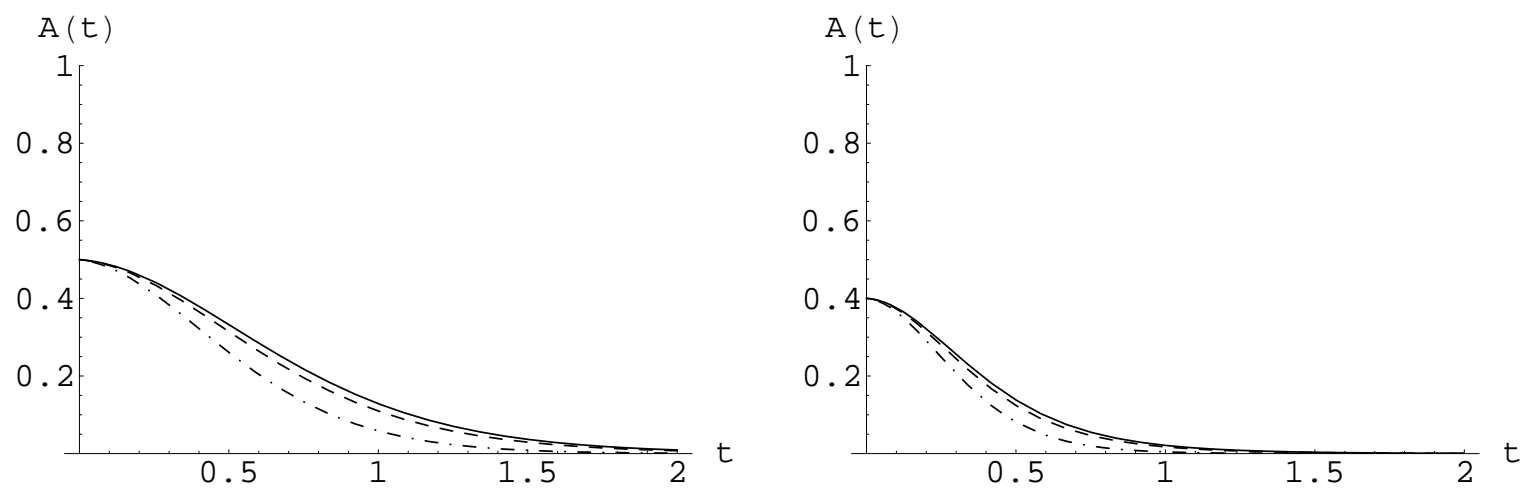

Figure 2: Left, anisotropy parameter $A$ for the geometrical Chaplygin gas filled Bianchi type I brane universe as a function of time and right, the same parameter in the Bianchi type $\mathrm{V}$ brane universe for $\gamma=4 / 3, \alpha=1$ and $A_{s}=0.3$ (solid line), $A_{s}=0.5$ (dashed line), $A_{s}=0.8$ (dot-dashed line) with $\Lambda=0$.
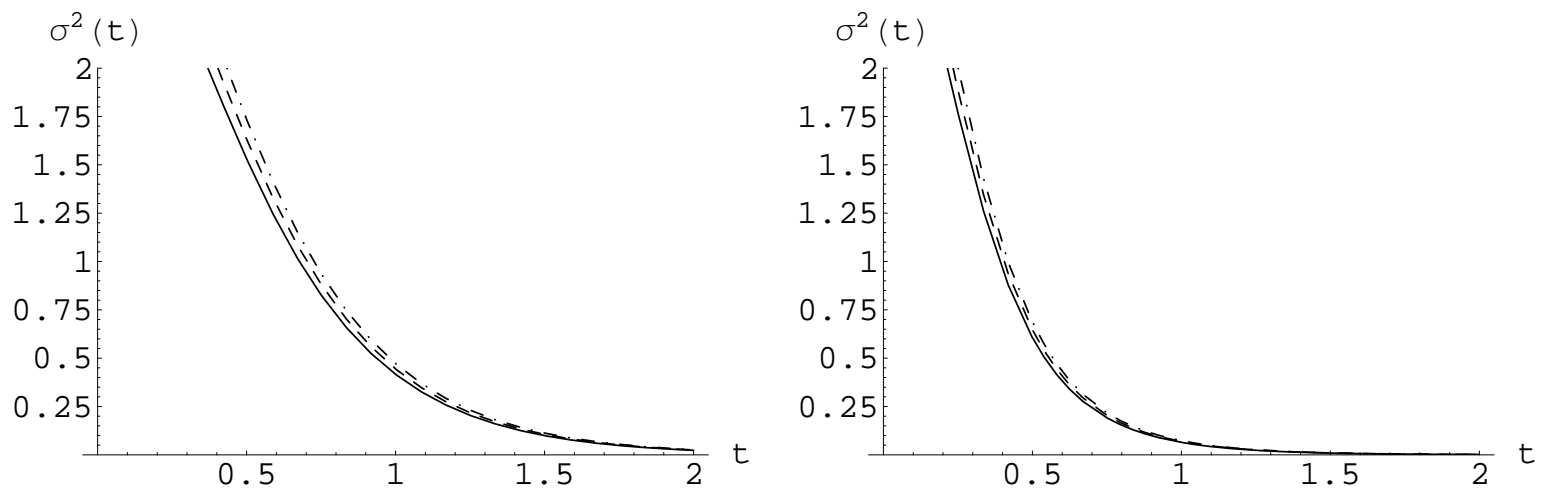

Figure 3: Left, shear scalar $\sigma^{2}$ for the geometrical Chaplygin gas filled Bianchi type I brane universe as a function of time and right, the same parameter in the Bianchi type $\mathrm{V}$ brane universe for $\gamma=4 / 3, \alpha=1$ and $A_{s}=0.3$ (solid line), $A_{s}=0.5$ (dashed line), $A_{s}=0.8$ (dot-dashed line) with $\Lambda=0$. 

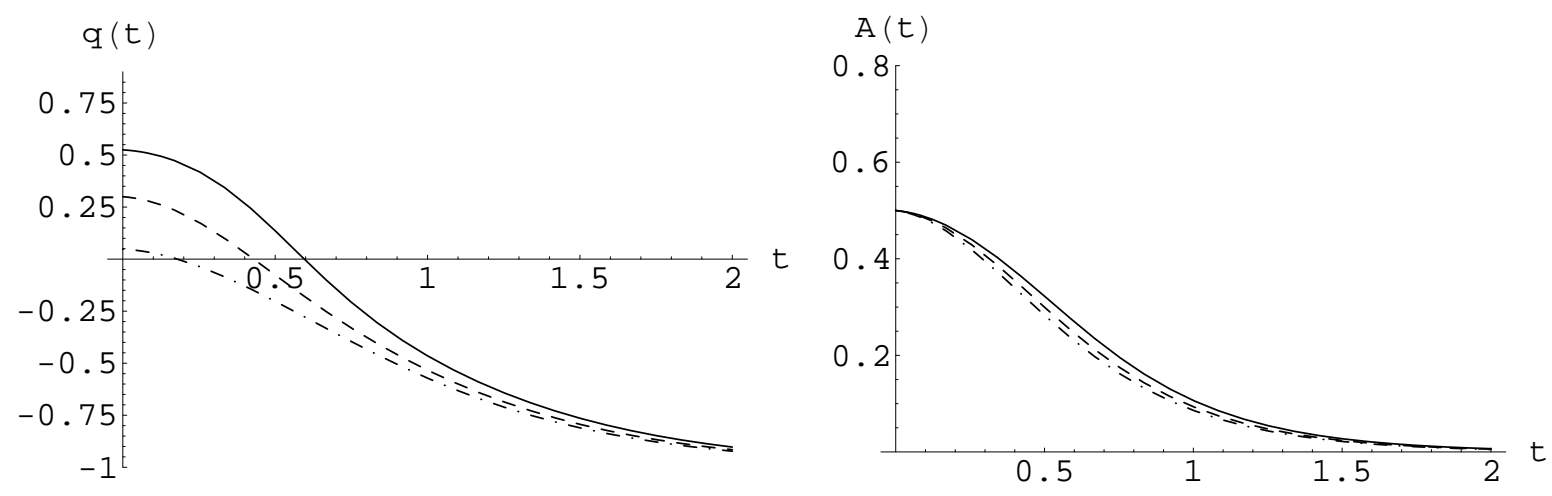

Figure 4: Left, deceleration parameter $q$ of the Bianchi type I brane universe as a function of time and right, anisotropy parameter $A$ of the Bianchi type I brane universe as a function of time for $\gamma=4 / 3, \alpha=3.75$ and $A_{s}=0.3$ (solid line), $A_{s}=0.6$ (dashed line), $A_{s}=0.936$ (dot-dashed line) with $\Lambda=0$.

The behavior of this parameter shows that the universe starts from a singular state with maximum anisotropy and ends up in an isotropic de Sitter inflationary phase at late times. The time variation of the shear parameter is represented for different values of $A_{s}$, in figure 3 .

It is worth mentioning that although most of the works on GCG cosmology have assumed a value for $\alpha$ compatible with $0<\alpha \leq 1$, it has been shown that the type-Ia Supernovae data favors $\alpha>1$ $[30,31,32]$. The best fitted values suggested for $\alpha>1$ are $\alpha=3.75$ and $A_{s}=0.936$ [30]. In figure 4 we have plotted the deceleration and anisotropy parameters of the Bianchi type I geometry for $\alpha=3.75$ and different values of $A_{s}$. This behavior shows that for $\alpha>1$ with $0 \leq A_{s} \leq 1$, the geometrical model presented in this work is in agreement with observational data.

\section{Conclusions}

In this paper we have shown that dark energy may be considered as a consequence of the extrinsic curvature in a brane-world scenario and extended the predictions of the geometrical matter in the more general case when the relation between $p$ and $\rho$ is not linear [26]. In this work, we have studied the Bianchi type I and V geometries, seen as a brane-world embedded in a five-dimensional bulk of constant curvature, without $Z_{2}$ symmetry or any form of junction condition. We have shown that the geometrical Chaplygin gas may be used to account for the accelerated expansion of an anisotropic universe. We have also obtained the general solutions in an exact parametric form for both Bianchi type I and V geometries and studied the behavior of the observationally important parameters.

The study of anisotropic homogeneous brane-world cosmological models has shown that in the framework of the RS models, brane universes are born into an isotropic state [11], whereas in the model presented here the universe starts as a singular state with maximum anisotropy and reaches an isotropic state in the late time limit, in agreement with the standard $4 D$ cosmology.

\section{References}

[1] A. Kamenshchik, U. Moschella and V. Pasquier, Phys. Lett. B 511265 (2001).

[2] M. C. Bento, O. Bertolami and A. A. Sen, Phys. Rev. D 66043507 (2002).

[3] N. Bilic, G. B. Tupper and R.D. Viollier, Phys. Lett. B 53517 (2002).

[4] A. Kamenshchik, U. Moschella and V. Pasquier, Phys. Lett. B 4877 (2000).

[5] S. K. Kama, Phys. Lett. B 42439 (1998). 
[6] J. C. Fabris, S. V. B. Goncalves and P. E. de Souza, Gen. Rel. Grav. 342111 (2002), M. Bordemann and J. Hoppe, Phys. Lett. B 317315 (1993),

N. Ogawa, Phys. Rev. D 62085023 (2000).

[7] M. C. Bento, O. Bertolami and A. A. Sen, Phys. Lett. B 575172 (2003).

[8] M. Makler, S. Q. de Oliveira and I. Waga, Phys. Lett. B 5551 (2003),

Y. Gong and C. K. Duan, Class. Quant. Grav. 213655 (2004).

[9] M. C. Bento, O. Bertolami and A. A. Sen, Phys. Lett. B 575172 (2003),

L. Amendola, F. Finelli, C. Burigana and D. Carturan, JCAP. 0307005 (2003),

M. C. Bento, O. Bertolami and A. A. Sen, Phys. Rev. D 67063003 (2003).

[10] R. Bean, O. Dore, Phys. Rev. D 68023515 (2003),

Z. H. Zhu, Astron. Astrophys. 423421 (2004).

[11] M. K. Mak and T. Harko, Phys. Rev. D 71104022 (2005).

[12] L. P. Chimento and R. Lazkoz, Phys. Lett. B 615146 (2005),

T. Barreiro, A. A. Sen, Phys. Rev. D 70124013 (2004),

P. F. Gonzalez-Diaz, Phys. Lett. B 5621 (2003),

L. P. Chimento, Phys. Rev. D 69123517 (2004),

G. M. Kremer, Phys. Rev. D 68123507 (2003),

C. S. J. Pun, L. A. Gergely, M. K. Mak, G. M. Szabo and T. Harko, Phys. Rev. D 77063528 (2008).

[13] P. Pedram and S. Jalalzadeh, Phys. Lett. B 6596 (2008),

U. Debnath, A. Banerjee and S. Chakraborty, Class. Quant. Grav. 215609 (2004),

M. C. Bento, O. Bertolami and A. A. Sen, Phys. Rev. D 70083519 (2004),

R. R. R. Reis, I. Waga, M. O. Calvao and S. E. Joras, Phys. Rev. D 68061302 (2003),

T. Multamaki, M. Manera and E. Gaztanaga, Phys. Rev. D 69023004 (2004),

P. P. Avelino, L. M. G. Beca, J. P. M. de Carvalho and C. J. A. P. Martins, JCAP 0309002 (2003).

[14] N. Arkani-Hamed, S. Dimopoulos, and G. Dvali, Phys. Lett. B 429263 (1998),

I. Antoniadis, N. Arkani-Hamed, S. Dimopoulos, and G. Dvali, Phys. Lett. B 436257 (1998).

[15] L. Randall and R. Sundrum, Phys. Rev. Lett. 833370 (1999),

L. Randall and R. Sundrum, Phys. Rev. Lett. 834690 (1999).

[16] G. Dvali, G. Gabadadze, and M. Porrati, Phys. Lett. B 485208 (2000),

G. Dvali and G. Gabadadze, Phys. Rev. D 63065007 (2001).

[17] W. Israel, Nuovo Cimento B 441 (1966).

[18] R. A. Battye and B. Carter, Phys. Lett. B 509331 (2001).

[19] J. M. Cline, C. Grojean and G. Servant, Phys. Rev. Lett. 834245 (1999),

T. Shiromizu, K. Maeda and M. Sasaki, Phys. Rev. D 62024012 (2000).

[20] P. Binetruy, C. Deffayet and D. Langlois, Nucl. Phys. B 565269 (2000),

P. Binetruy, C. Deffayet, U. Ellwanger and D. Langlois, Phys. Lett. B 477285 (2000).

[21] M. D. Maia, E. M. Monte and J. M. F. Maia, Phys. Lett. B 58511 (2004).

[22] M. D. Maia, E. M. Monte, J. M. F. Maia and J. S. Alcaniz, Class. Quant. Grav. 221623 (2005).

[23] V. A. Rubakov and M. E. Shaposhnikov, Phys. Lett. B 125136 (1983). 
[24] S. Jalazadeh and H. R. Sepangi, Class. Quant. Grav. 222035 (2005).

[25] M. Heydari-Fard, M. Shirazi, S. Jalalzadeh and H. R. Sepangi, Phys. Lett. B 6401 (2006).

[26] M. Heydari-Fard and H. R. Sepangi, Phys. Rev. D 76104009 (2007).

[27] J. Nash, Ann. Maths. 6320 (1956).

[28] L. P. Eisenhart 1966 Riemannian Geometry, Princeton University Press, Princeton NJ (1966).

[29] C. M. Chen, T. Harko and M. K. Mak, Phys. Rev. D 64044013 (2001).

[30] O. Bertolami, A. A. Sen, S. Sen and P. T. Silva, Mon. Not. Roy. Astron. Soc. 353329 (2004).

[31] M. C. Bento, O. Bertolami, N. M. C. Santos and A. A. Sen, Phys. Rev. D 71063501 (2005).

[32] M. C. Bento, O. Bertolami, M. J. Rebouas and P. T. Silva, Phys. Rev. D 73043504 (2006), A. A. Sen, R. J. Scherrer, Phys. Rev. D 72063511 (2005), Y. Gong, JCAP 0503007 (2005). 